\title{
mRNA level of ROCK1, RHOA, and LIMK2 as genes associated with apoptosis in evaluation of effectiveness of adalimumab treatment
}

\author{
Agata Krawczyk ${ }^{1} \cdot$ Barbara Strzałka-Mrozik ${ }^{2} \cdot$ Beniamin Grabarek $^{3,4} \cdot$ Dominika Wcisło-Dziadecka $^{5}$. \\ Magdalena Kimsa-Dudek ${ }^{1} \cdot$ Celina Kruszniewska-Rajs ${ }^{2} \cdot$ Joanna Gola $^{2}$
}

Received: 7 August 2019 / Revised: 11 November 2019 / Accepted: 29 November 2019 / Published online: 2 March 2020

(c) The Author(s) 2020

\begin{abstract}
Background Psoriasis is a multifactorial autoimmune disease, which underlies the abnormalities of the apoptotic process. In cases of psoriasis and psoriatic arthritis, biological treatment is used. This study aimed to determine any changes in the expression of the genes associated with apoptosis in patients with psoriatic arthritis treated with adalimumab and to assess any phenotypic modifications based on changes in dermatological indexes.

Methods The study included 20 patients with psoriatic arthritis treated biologically and 20 healthy volunteers. The research material consisted of peripheral blood mononuclear cells (PBMCs) from which the total RNA was isolated. Changes in the gene expression were determined using oligonucleotide microarrays and RT-qPCR. The clinical condition was assessed based on selected indicators: PASI, BSA [\%], DAS28, and DLQI, which were determined every 3 months.

Results There were changes in the expression of genes associated with apoptosis. Significant differences were found for ROCK1, RhoA, and LIMK2 expression profiles in PBMCs. At the initial stage of treatment, a decrease in the PASI and BSA rates was observed. At the later stages, the values of these indicators increased once again. There were correlations between the changes in these genes' expression and the dermatological markers.

Conclusion Adalimumab influences the expression of genes related to apoptosis and the values of dermatological indicators of patients. Changes in the expression level of genes associated with apoptosis suggest that ROCK1, RhoA, and LIMK2 may be genes that can potentially be indicators of treatment effectiveness and lack of response to biological treatment.
\end{abstract}

Keywords Psoriatic arthritis $\cdot$ Apoptosis $\cdot R O C K \cdot$ Drug resistance $\cdot$ Adalimumab

Agata Krawczyk

akrawczyk@sum.edu.pl

1 Department of Nutrigenomics and Bromatology, Faculty of Pharmaceutical Sciences in Sosnowiec, Medical University of Silesia in Katowice, Jednosci 8 Street, 41-200 Sosnowiec, Poland

2 Department of Molecular Biology, Faculty of Pharmaceutical Sciences in Sosnowiec, Medical University of Silesia in Katowice, Sosnowiec, Poland

3 Department of Histology, Cytophysiology and Embryology, Faculty of Medicine in Zabrze, University of Technology in Katowice, Zabrze, Poland

4 Department of Clinical Trials, Maria Sklodowska-Curie National Research Institute of Oncology Krakow Branch, Krakow, Poland

5 Department of Cosmetology, Faculty of Pharmaceutical Sciences in Sosnowiec, Medical University of Silesia in Katowice, Sosnowiec, Poland

\section{Introduction}

Apoptosis is an active physiological process during which a pathway that induces programmed cell death is triggered as a result of the stimulation. This process involves individual cells characterized by a lack of inflammation. At the first stage of apoptosis, the genetic material in the nucleus of a cell undergoes condensation. The cell shrinks and the cytoskeleton breaks down. Cell organelles remain intact. Thus, cell membrane continuity also remains intact. Apoptotic bodies are formed. Finally, there is phagocytosis in which the macrophages and keratinocytes are primarily involved. The recognizable characteristic of this phase of cell death is the phospholipid asymmetry and the phosphatidylserine shift outside the cell membrane. This process is involved in removing damaged cells or cell fragments. It takes part in defense processes [1]. 
There are two pathways of apoptosis-extrinsic and intrinsic. The extrinsic pathway (receptor pathway) is induced when a membrane death receptor binds to the corresponding ligand, resulting in the activation of a caspase cascade. The most characteristic and best known example is tumor necrosis factor receptor 1 (TNFR1) [2].

The intrinsic pathway, which is called the mitochondrial pathway, is activated by, for example, an increase in the concentration of free radicals (FRs) in a cell and its surroundings as well as DNA damage. As a result of a decrease in the mitochondrial membrane potential, cytochrome $\mathrm{c}$ is introduced into the cytoplasm, after which a caspase-9-mediated pathway is activated. The anti-apoptotic Bcl-2 family of proteins regulates this type of path. Bid, Bad, Bak, and Bax are examples of pro-apoptotic proteins [3].

Apoptosis is a critical process for various disease entities, such as cancer [4], autoimmune disease [5, 6], dermatological diseases (scleroderma, lichen planus), and AIDS [7]. In the case of psoriasis, which is classified as an inflammatory autoimmune disease, regulating the keratinocyte proliferation process depends on the balance between the expression of the pro- and anti-apoptotic proteins. This is the case in respect to this specific type of apoptosis. This leads to keratinocyte proliferation that is caused by the replacement of the contents of a cell by cytoskeletal proteins, after which the epidermis and its components undergo hypertrophy. It should be noted that diseases that are characterized by an inhibition of keratinocyte apoptosis are chronic. A reduced percentage of apoptotic cells are observed in the psoriatic epidermis compared to the healthy epidermis. Also, keratinocytes from psoriatic lesions are more resistant to apoptosis-inducing factors compared to normal cells [8].

In psoriasis and psoriatic arthritis, there is a close and inseparable connection between a disturbance in the correct level and the rate of the apoptosis of the immune cells. This is characterized by the ability to migrate to the settled cells of the epidermis, thus creating a microenvironment. Lymphocytes, whose survival time is prolonged, most likely cause the chronic course of a disease, resistance to treatment, and numerous relapses [9]. The correct course of the apoptotic process determines the maintenance of homeostasis within the epidermis and also ensures the proper functioning of the immune system [3].

It has been reported that the concentration of tumor necrosis factor $\alpha(\mathrm{TNF}-\alpha)$ is increased in serum in patients with psoriasis. Biopsies have also revealed the increased concentration of TNF- $\alpha$, which, considering the pro-apoptotic properties of TNF- $\alpha$, is kind of a paradox. This is caused by the resistance of psoriatic skin keratinocytes to the signals that are transmitted through the interaction of this cytokine with its receptors [1]. This phenomenon explains the legitimacy of the implementation of biological therapy against TNF- $\alpha$ in treating psoriasis. The biological drugs that are
TNF- $\alpha$ inhibitors include adalimumab, which is an exclusively human monoclonal antibody [10]. The mechanism of action of the drug is dependent on its specific binding to TNF, which neutralizes its biological activity by blocking its interaction with surface receptors TNFR1 and TNFR2. Thus, this drug can work during various stages of signal transduction [11]. Other types of biological medicines used to treat psoriasis and psoriatic arthritis are anti IL-17 and anti IL-12/23 drugs. Despite the large number of positive effects of these drugs (initial improvement of patients' clinical condition and reduction of the incidence of vascular disease symptoms), numerous negative effects have also been noted [12]. It is worth stating that therapy using biological drugs from the anti-TNF group does not always bring satisfactory therapeutic effects. It has been proven that, in patients not responding to anti-TNF treatment, high therapeutic efficacy was achieved using IL-17 neutralizing drugs [13]. However, one possible undesirable effect would be drug resistance. An example of this occurs with the drug etanercept [14].

This study aimed to determine any changes in the gene expression that are connected with apoptosis in patients with psoriatic arthritis who are being treated with adalimumab compared with healthy volunteers (control). Changes in the values of the dermatological indexes, Psoriasis Area and Severity Index (PASI), body surface area (BSA), Dermatology Life Quality Index (DLQI), and Disease Activity Score 28 (DAS28), were used to analyze the effectiveness of the treatment at the phenotypic level.

\section{Materials and methods}

\section{Test and control groups}

The study involved 20 patients ( 12 men and 8 women; mean age of $48 \pm 10$ years) with diagnosed psoriatic arthritis in the Polish population who were being treated with adalimumab. The patients were administered a $40 \mathrm{mg}$ dose every 2 weeks for 24 months. Patients were not given any other dermatological drugs. Before the study, patients were treated with cyclosporin A, methotrexate, or sulfasalazine. Every 3 months, there were follow-up visits, which were defined as monitoring $[\mathrm{M}]$ in the later part of the study, during which any changes in the dermatological indexes [PASI, BSA (\%), DLQI, and DAS28] were determined and venous blood was collected for molecular analysis.

The inclusion and exclusion criteria that are set out in the recommendations of the Polish Dermatological Association regarding the use of biological drugs in psoriasis and psoriatic arthritis were followed [15].

The control group consisted of 20 people (12 men and 8 women; mean age of $46 \pm 10$ years). The inclusion criterion was the lack of dermatological diseases for at least 3 months, 
during which time the volunteers did not use general steroid therapy. The criteria for exclusion from the study included alcohol abuse, pregnancy, breastfeeding, and immunosuppressive treatment (during the previous 3 months).

The approval of the Bioethical Commission of the Medical University of Silesia in Katowice (KNW/0022/ $\mathrm{KB} 1 / 59 / 13$ ) was obtained for the tests in accordance with the Declaration of Helsinki on human medical research. The patients who were included in the study indicated their agreement to participate in the project by signing consent form.

\section{Test material}

The test materials were PBMCs, which were obtained from whole blood from the patients and healthy volunteers (control). The material for the molecular analyses was collected before the treatment with adalimumab [M0], and then every 3 months immediately before the next dose of the TNF- $\alpha$ inhibitor [M1-8] was administered. Blood was only collected once from the healthy volunteers.

\section{Total RNA extraction}

The molecular analyses included the extraction of total RNA from the PBMCs using a set of RNA isolation reagents-a PAXgene Blood RNA Kit IVD (Qiagen, Wrocław, Poland)—according to the manufacturer's instructions. A Nano MN-913 nanospectrophotometer (Maestrogen, Hsinchu City, Taiwan) was used to assess nucleic acid concentration. The products of the individual stages were also qualitatively assessed in a $1 \%$ agarose gel using the electrophoresis technique. The total RNA was used as the template for transcriptomes that were to be determined using the microarray method.

\section{Analysis of the gene expression profile using the oligonucleotide microarray technique}

The gene expression profile in the tested material was analyzed using the oligonucleotide microarray HG-U 133A 2.0 (Affymetrix, Santa Clara, CA, USA) according to the manufacturer's recommendations. The procedure for determining the transcriptomes using the oligonucleotide microarray technique included the following steps: synthesizing the cDNA; synthesizing, labeling, and fragmenting the cRNA; hybridizing the samples using probes; reading the fluorescence signal; and analyzing the data.

\section{RT-qPCR reaction in real time}

Real-time quantitative reverse transcription polymerase chain reaction (RT-qPCR) was performed for the genes ROCK1, RhoA, and LIMK2 with ACTB as the endogenous control according to Sahlol et al. [16] and Arul et al. [17] (Table 1). Quantitative analysis was performed using a SensiFAST ${ }^{\mathrm{TM}}$ SYBR No-ROX One-Step Kit (Bioline, London, UK) according to the manufacturer's instructions and the DNA Engine Opticon ${ }^{\mathrm{TM}}$ System (MJ Research Inc., Waltham, MA, USA). The RT-qPCR reaction for each of the analyzed genes was performed in three technical repetitions. The number of mRNA copies was converted to $1 \mu \mathrm{g}$ of the total RNA.

\section{Statistical analysis}

The results that were obtained by oligonucleotide microarrays were analyzed using the GeneSpring 13.0 platform (Agilent Technologies, UK Limited, South Queensferry, UK) and the PL-Grid platform (https://www.plgrid.pl/). A list of genes was prepared, the differential expressions of which were determined at the level of at least a twofold change in at least one pair at the significance level of $p<0.05$. Any significant differences in gene expression between the study group and the control group were assessed using the one-way analysis of variance (ANOVA) test with
Table 1 Characteristics of the primers that were used for the real-time RT-qPCR

\begin{tabular}{|c|c|c|c|}
\hline Gene & Primer sequence & $\begin{array}{l}\text { Amplicon } \\
\text { length (bp) }\end{array}$ & $T_{\mathrm{m}}\left({ }^{\circ} \mathrm{C}\right)$ \\
\hline$R O C K 1$ & $\begin{array}{l}\text { Forward: 5'-CAAGGTGGTGATGGTTATTATG-3' } \\
\text { Reverse: 5'-TATCACCTACAAGCATTTCG-3' }\end{array}$ & 103 & 77.8 \\
\hline RhoA & $\begin{array}{l}\text { Forward: 5'-AGGAAGATTATGATCGCCTG-3' } \\
\text { Reverse: 5'-TTCTAAACTATCAGGGCTGTC-3' }\end{array}$ & 92 & 79.4 \\
\hline$L I M K 2$ & $\begin{array}{l}\text { Forward: 5'-TATCAAGGTGACACACAAAG-3' } \\
\text { Reverse: 5'-CTTTCACCTCAGTCAGAAAAG-5' }\end{array}$ & 101 & 79.8 \\
\hline$A C T B$ & $\begin{array}{l}\text { Forward: 5'-TCACCCACACTGTGCC CATCTACGA-3' } \\
\text { Reverse: 5'-CAGCGGAACCGCTCATTGCCAATGG-3' }\end{array}$ & 295 & 87.6 \\
\hline
\end{tabular}

$b p$ base pairs, $T_{m}$ melting temperature 
the Benjamini-Hochberg correction. The results that were obtained using RT-qPCR in real time were analyzed statistically with Statistica 13.3 software (StatSoft, Kraków, Poland) using the non-parametric Friedman's ANOVA test and its post hoc test (equivalent to Tukey's test). The values that were obtained using RT-qPCR in real time were expressed as the median of the 25 th and 75 th quartiles. The relationship between adalimumab intake and the dermatological index values were assessed using an ANOVA analysis with repeated measures, which was followed by Tukey's post hoc test $(p<0.05)$. Spearman rank order correlation $\left(r_{\mathrm{s}}\right)$ was used to evaluate the relationships indicated by the data, and the results were considered to be statistically significant when the significance level was $p<0.05$.

\section{Results}

At the first stage of the research, the oligonucleotide microarray HG-U 133A 2.0 (Affymetrix) genes that were connected with the process of apoptosis and that differentiated the examined groups were determined. Genes were selected from the NetAffx2 Analysis Center database of Affymetrix (https://www.affymetrix.com/analysis/index.affx; access date: August 2018). Among the 334 mRNA IDs that were involved in the apoptosis process, the mRNA IDs that were identified using the one-way ANOVA analysis was statistically significant $(p<0.05)$.

Then, using the multiple comparison test (post hoc Tukey's test) at the significance level of $p<0.05$, the number of mRNA IDs that differentiated the examined transcripts was determined. Before the first dose of [M0] vs. the control [C], 94 mRNA IDs were selected, and before the next dose of the drug after 3 months of treatment with [M1] (after 3 months of therapy) vs. [C], 95 mRNA IDs were selected, while for [M0] vs. [M1] (after 3 months of treatment), one differentiation mRNA ID was selected.

Overall, 50 mRNA IDs that had more than a twofold statistically significant change in expression in at least one pair were determined in this analysis (Table 2).

The next step in the analysis was to assess the specificity of mRNA IDs in differentiating the analyzed transcriptome groups in the individual groups of subjects based on the results that were obtained with Tukey's post hoc test using a Venn diagram (Fig. 1).

The RT-qPCR reaction was performed for $R O C K 1$, which differentiated [M1] vs. [M0]. The RhoA and LIMK2 genes were also analyzed (selected based on the literature) to monitor the treatment with adalimumab using gene expression analysis $[18,19]$.

These analyses determined that there was a statistically significant change in the expression of the ROCKI gene $(p=0.0007)$ during adalimumab therapy. The RT-qPCR analyses confirmed the direction of the expression changes for these gene transcripts. A decrease in the ROCK1 gene expression was observed for the comparison of [M0] vs. [C] and [M1] vs. [C], while the expression of [M1] vs. [M0] was at the same level. An increase in its expression was observed for [M2] vs. [M0]. This trend continued until the 15th month of the anti-TNF treatment [M5], after which the expression of the ROCK1 gene decreased once again. Further analyses using Tukey's post hoc multiple comparison test showed statistically significant changes in gene expression for [M0] vs. [C] $(p=0.0086) ; 12$ months of treatment with [M4] vs. [M1] ( $p=0.0476)$; and [M4] vs. [M0] ( $p=0.0476)$ (Fig. 2a).

There were also statistically significant changes in the expression profile of the RhoA genes $(p=0.0266)$ and LIMK2 $(p=0.0105)$. There were statistically significant changes in the expression of the RhoA gene between the $21 \mathrm{st}$ month of therapy for [M7] vs. [M0] ( $p=0.0276)$ (Fig. 2b). Moreover, for the LIMK2 gene, there was a statistical significance in the number of copies of mRNA/ $\mu \mathrm{g}$ of total RNA for [M4] vs. [M1] $(p=0.0373)$ (Fig. 2c).

The clinical picture of the patients changed with the next doses of the drug. There was a decrease in the value of the dermatological indicators of PASI and BSA [\%]. The downward trend continued in [M3] for the PASI indicator and to [M2] for the BSA [\%] indicator. During the next monitoring, the value of these indicators increased. In [M5], the PASI indicator fell again, and then in the subsequent control, it balanced at similar levels. An increase in BSA [\%] was recorded in [M3], followed by a decrease in [M5]. In subsequent monitoring, the indicator remained at similar levels. Similar results were found for the DLQI and DAS 28 indicators (Table 3).

There were statistically significant $(p<0.05)$ correlations $\left(r_{s}\right)$ between DLQI and changes in the level of the RhoA gene expression in [M0] $\left(r_{\mathrm{s}}=-0.82, p=0.02\right)$, and the BSA index and the ROCK1 gene expression level at the same monitoring time $\left(r_{\mathrm{s}}=-0.84, p=0.04\right)$. During [M2], statistically significant correlations between the BSA index and the level of the ROCK 1 gene expression were observed $\left(r_{\mathrm{s}}=-0.80\right.$, $p=0.03$ ). In the case of [M4], a correlation occurred between the PASI index and the LIMK2 gene expression $\left(r_{\mathrm{s}}=0.88, p=0.02\right)$. Correlations between DAS28 and the $R O C K 1$ gene expression $\left(r_{\mathrm{s}}=0.99, p=0.007\right)$ and the DLQI index and the RhoA gene expression were recorded in [M5], while in [M7], a correlation was observed for the BSA index and the ROCKI gene expression $\left(r_{\mathrm{s}}=0.98 ; p=0.02\right)$ and the PASI index and the ROCK1 gene expression $\left(r_{\mathrm{s}}=0.98\right.$, $p=0.02$ ). 
Table 2 List of the transcripts that are connected with apoptosis, which had more than a twofold statistically significant change in their expression in at least one pair

\begin{tabular}{|c|c|c|c|c|}
\hline ID & Gene symbol & FC ([M0] vs. [C] $)$ & FC ([M1] vs. [C] $)$ & FC ([M1] vs. [M0] \\
\hline 200679_x_at & HMGBI & $\downarrow 2,02$ & $\downarrow 1,97$ & $\uparrow 1,02$ \\
\hline 201502_s_at & NFKBIA & $\downarrow 2,01$ & $\downarrow 2,14$ & $\downarrow 1,06$ \\
\hline 201715_s_at & ACINI & $\downarrow 1,97$ & $\downarrow 2,11$ & $\downarrow 1,07$ \\
\hline 201746_at & TP53 & $\downarrow 3,60$ & $\downarrow 3,83$ & $\downarrow 1,06$ \\
\hline 202597_at & IRF6 & $\downarrow 2,20$ & $\downarrow 2,18$ & $\uparrow 1,01$ \\
\hline 202621_at & IRF3 & $\downarrow 5,97$ & $\downarrow 6,56$ & $\downarrow 1,10$ \\
\hline 202687_s_at & TNFSF10 & $\uparrow 4,27$ & $\uparrow 4,79$ & $\uparrow 1,12$ \\
\hline 202688_at & TNFSF10 & $\uparrow 1,82$ & $\uparrow 2,07$ & $\uparrow 1,14$ \\
\hline 204780_s_at & FAS & $\uparrow 2,41$ & $\uparrow 3,02$ & $\uparrow 1,25$ \\
\hline 205468_s_at & IRF5 & $\downarrow 2,80$ & $\downarrow 2,77$ & $\uparrow 1,01$ \\
\hline 205599_at & TRAFI & $\downarrow 1,91$ & $\downarrow 2,11$ & $\downarrow 1,10$ \\
\hline 206665_s_at & $B C L 2 L 1$ & $\downarrow 1,91$ & $\downarrow 2,86$ & $\downarrow 1,05$ \\
\hline 207163_s_at & $A K T I$ & $\downarrow 2,66$ & $\downarrow 2,75$ & $\downarrow 1,04$ \\
\hline 207163_s_at & PTK2 & $\downarrow 2,07$ & $\downarrow 2,30$ & $\downarrow 1,11$ \\
\hline 208611_s_at & SPTAN1 & $\downarrow 5,46$ & $\downarrow 5,19$ & $\uparrow 1,05$ \\
\hline 209310_s_at & CASP4 & $\uparrow 2,04$ & $\uparrow 2,55$ & $\uparrow 1,25$ \\
\hline 209508_x_at & CFLAR & $\uparrow 1,83$ & $\uparrow 2,09$ & $\uparrow 1,14$ \\
\hline 209790_s_at & CASP6 & $\uparrow 3,00$ & $\uparrow 2,77$ & $\downarrow 1,08$ \\
\hline 209811_at & CASP2 & $\uparrow 6,05$ & $\uparrow 4,97$ & $\downarrow 1,22$ \\
\hline 209831_x_at & DNASE2 & $\uparrow 1,87$ & $\uparrow 2,05$ & $\uparrow 1,10$ \\
\hline 209878_s_at & RELA & $\downarrow 2,91$ & $\downarrow 2,77$ & $\uparrow 1,05$ \\
\hline 209929_s_at & $I K B K G$ & $\downarrow 2,00$ & $\downarrow 1,99$ & $\uparrow 1,01$ \\
\hline 211027_s_at & $I K B K B$ & $\downarrow 3,89$ & $\downarrow 4,47$ & $\downarrow 1,15$ \\
\hline 211282_x_at & TNFRSF 25 & $\downarrow 4,84$ & $\downarrow 4,86$ & $\downarrow 1,00$ \\
\hline 211300 _s_at & TP53 & $\downarrow 8,85$ & $\downarrow 8,39$ & $\uparrow 1,06$ \\
\hline 211316_x_at & CFLAR & $\uparrow 2,01$ & $\uparrow 2,25$ & $\uparrow 1,12$ \\
\hline 211822_s_at & NLRPI & $\downarrow 3,27$ & $\downarrow 3,43$ & $\downarrow 1,05$ \\
\hline 211824_x_at & NLRPI & $\downarrow 3,45$ & $\downarrow 3,48$ & $\downarrow 1,01$ \\
\hline 211841_s_at & TNFRSF 25 & $\downarrow 2,57$ & $\downarrow 2,80$ & $\downarrow 1,09$ \\
\hline 213105_s_at & TSR3 & $\uparrow 2,14$ & $\uparrow 2,08$ & $\downarrow 1,03$ \\
\hline 214329_x_at & TNFSF 10 & $\uparrow 2,54$ & $\uparrow 2,98$ & $\uparrow 1,17$ \\
\hline 214617_at & PRF1 & $\downarrow 2,45$ & $\downarrow 2,47$ & $\downarrow 1,01$ \\
\hline 214726_x_at & $A D D 1$ & $\downarrow 3,54$ & $\downarrow 3,79$ & $\downarrow 1,07$ \\
\hline 214736_s_at & $A D D 1$ & $\downarrow 2,21$ & $\downarrow 2,12$ & $\uparrow 1,04$ \\
\hline w214786_at & $M A P 3 K I$ & $\downarrow 2,48$ & $\downarrow 2,31$ & $\uparrow 1,07$ \\
\hline 214925_s_at & SPTANI & $\downarrow 1,96$ & $\downarrow 2,56$ & $\downarrow 1,31$ \\
\hline 215037 s at & $B C L 2 L 1$ & $\downarrow 4,34$ & $\downarrow 4,57$ & $\downarrow 1,05$ \\
\hline 215235_at & SPTANI & $\downarrow 2,61$ & $\downarrow 2,58$ & $\uparrow 1,01$ \\
\hline 215310_at & $A P C$ & $\uparrow 2,56$ & $\uparrow 2,38$ & $\downarrow 1,07$ \\
\hline 216971_s_at & PLEC & $\downarrow 6,93$ & $\downarrow 7,7$ & $\downarrow 1,03$ \\
\hline 218056 at & $B F A R$ & $\uparrow 2,08$ & $\uparrow 1,90$ & $\downarrow 1,09$ \\
\hline 219333_s_at & CAPNIO & $\downarrow 2,58$ & $\downarrow 2,59$ & $\downarrow 1,01$ \\
\hline 219423_x_at & TNFRSF 25 & $\downarrow 2,19$ & $\downarrow 2,49$ & $\downarrow 1,14$ \\
\hline 221479_s_at & $B N I P 3 L$ & $\uparrow 5,78$ & $\uparrow 5,19$ & $\downarrow 1,12$ \\
\hline 221601_s_at & FAIM3 & $\downarrow 2,21$ & $\downarrow 2,54$ & $\downarrow 1,15$ \\
\hline 221602_s_at & FAIM3 & $\downarrow 5,07$ & $\downarrow 5,59$ & $\downarrow 1,10$ \\
\hline 36004_at & IKBKG & $\downarrow 2,14$ & $\downarrow 2,36$ & $\uparrow 1,11$ \\
\hline 37028_at & PPPIR15A & $\downarrow 2,78$ & $\downarrow 2,61$ & $\uparrow 1,06$ \\
\hline 206464_at & $B M X$ & $\uparrow 1,91$ & $\uparrow 2,87$ & $\uparrow 1,50$ \\
\hline 214578_s_at & ROCKI & $\downarrow 2,94$ & $\downarrow 1,45$ & $\uparrow 1,34$ \\
\hline
\end{tabular}

[C] control, [MO] prior to receiving the first dose of the drug, [M1] prior to receiving the next dose after 3 months of treatment, $[F C]$ fold change, $\mathrm{FC} \geq 2.0$, $\uparrow$ overexpression of a gene (increased level of mRNA IDs), $\downarrow$ suppressed gene expression (decreased level of mRNA IDs), ID the probe set identifier; the box highlighted in light gray: the transcript that differentiated the peripheral blood mononuclear cells (PBMCs) of patients [M1] vs. [C]; the box marked in dark gray: the transcript that differentiated the PBMCs of patients [M1] vs. [M0] 
Fig. 1 Venn diagram grouping the probe set identifiers (mRNA IDs) that were selected using Tukey's post hoc test with the Benjamini-Hochberg correction (after the one-way ANOVA test), depending on the specificity of the different groups of peripheral blood mononuclear cells (PBMCs) transcripts. [C] control, [MO] before receiving the first dose of the drug, [MI] before receiving the next dose of the medicine after 3 months, $[F C]$ fold change, $(\mathrm{FC}) \geq 2.0$; the box highlighted in light gray: the transcript that differentiated the PBMCs of patients [M1] vs. [C]; the box marked in dark gray: the transcript that differentiated the PBMCs of patients [M1] vs. [M0]
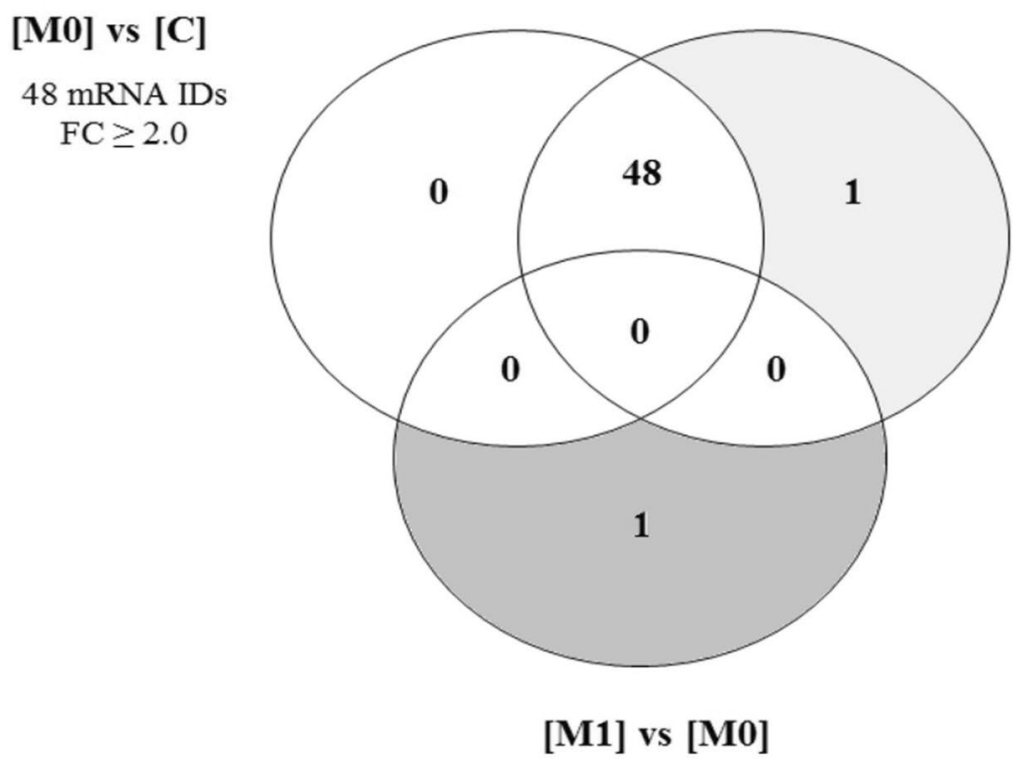

[M1] vs [C]

49 mRNA IDs

$\mathrm{FC} \geq 2.0$

\section{Discussion}

Analyzing transcriptomes using molecular biology techniques is a crucial aspect in assessing the effectiveness of therapy and analyzing any changes in the cellular or biological processes that occur during anti-cytokine treatment.

Because of the dynamic development of molecular techniques, it has become possible to identify the gene transcripts that could act as new complementary molecular markers for detecting a lack of response to treatment as well as for deciding to change the therapy before adverse phenotypic changes occur [20-22]. This is because molecular changes are preceded by phenotypic changes [23] and the molecular marker systems entirely fit the strategy of personalized diagnostics and treatment $[24,25]$.

One of the critical processes whose disorder is observed in the course of psoriasis is the loss of the balance between the process of apoptosis and cell proliferation [3, 24]. As part of this work, we decided to evaluate the effect of adalimumab therapy on the gene expression profile that is involved in apoptosis in patients with psoriatic arthritis and in healthy volunteers. For the patients with psoriasis, the values of the selected dermatological indexes (PASI, BSA, DAS28, and DLQI), for which there was a dependence on changes in the expression profile of the key analyzed genes, were evaluated during each visit. Twenty subjects in the study group and 20 healthy volunteers were assessed, which may seem to be relatively small samples. It should be noted, however, that the inclusion and exclusion criteria for each group were rigorous. The reason for this is that, according to the recommendations of the Polish Dermatological
Society for anti-cytokine therapy (including adalimumab), only patients with moderate to severe psoriasis are eligible to participate in studies. Moreover, no adequate clinical response to conventional treatment has been observed [26, 27]. Also, the efficacy of adalimumab in treating psoriatic arthritis at the molecular and clinical levels for 24 months was analyzed, and performing a microarray analysis was the first step of an in-depth molecular evaluation.

The first stage of our analysis allowed us to confirm the difference in the patterns of the expression of 94 gene transcripts that are involved in apoptosis between the patients who had qualified for anti-TNF therapy and the healthy volunteers. The results were consistent with those of Batiwalla et al. who emphasized the value of profiling the microarray PBMCs that are obtained from patients with psoriasis and healthy individuals. Moreover, they found that the PBMCs indicated that the gene transcripts encoding the proteins that are associated with apoptosis may constitute a promising pool of genes that differentiate not only healthy patients but also patients with ordinary articular psoriasis [28].

In our work, the following genes associated with apoptosis were analyzed for changes in their mRNAs level: ROCK1, $R h o A$, and LIMK2. These genes are closely related to many levels. RhoA activates certain effector proteins, mainly ROCK 1 and ROCK2. One of the substrates of ROCK's kinases is Ser/Thr LIM kinase (LIMK1 and LIMK2) [29]. Ren et al. [30] described a close and inseparable relationship among the three protein products that are encoded by these three genes in respect to neoplastic transformation and autophagy. They found that a discontinuation of the possibility of biological activity by ROCK1, RhoA, and LIMK2 

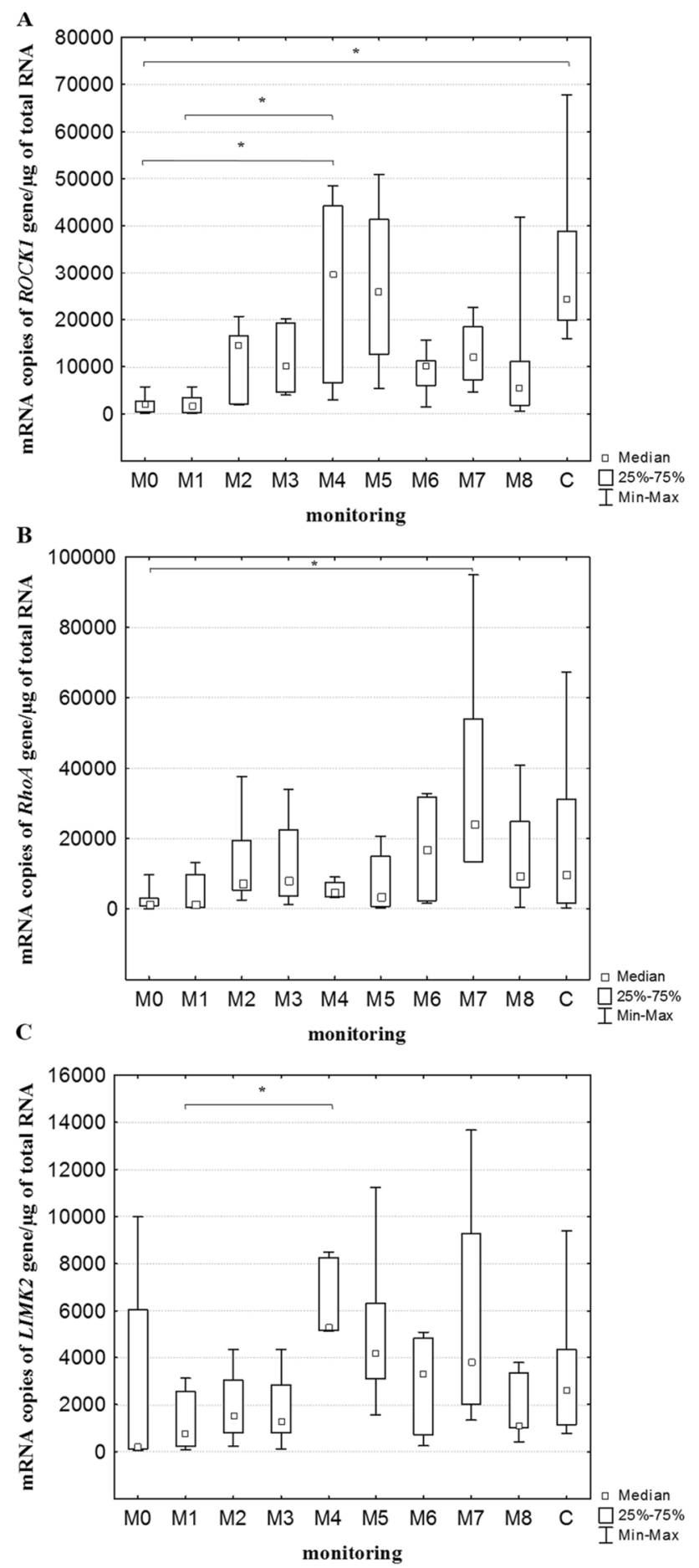

Fig. 2 The number of copies of mRNA/ $\mu$ g of total RNA: ROCK1 (a), RhoA (b) and LIMK2 (c) in the peripheral blood mononuclear cells (PBMCs) of the psoriatic patients who were being treated with adalimumab during eight time periods every 3 months. $[M]$ monitoring. Box and whisker plots present medians \pm quartiles and extreme values of copy numbers per $1 \mu \mathrm{g}$ of total RNA. *Statistical significance $p<0.05$, Friedman ANOVA test

contributes to the suppression of autophagy and the silencing of the carcinogenesis process. The 3-month adalimumab therapy showed that the ROCK1 gene is silenced almost threefold, but that under the influence of adalimumab therapy, there was a gradual increase in the expression of this gene to values close to those in control. This confirms that the transcripts are a dynamic system that reacts quickly to changes in the environment. This gene belongs to the family of genes encoding the $R O C K$ proteins, whose role is primarily described in the context of proliferation, differentiation, and cytoskeleton reorganization [31]. It seems, therefore, that the aberrant expression of ROCK1 may not only interfere with the balance between cell proliferation and apoptosis but, at the same time, may influence changes in their phenotype, thus leading to their differentiation towards other types of cells. Benitah et al. and Wilkinson et al. indicated that the ROCK1 and ROCK2 expression plays an important role in cancer progression and that these two genes may be responsible for the acquisition of metastatic potential by tumor cells [32, 33]. This confirms our previous observations on an in vitro model of dermal fibroblasts that had been exposed to adalimumab. We observed a change in the expression of the $B I R C$ gene coding for survivin, which is a protein whose detection is usually not observed under physiological conditions [34], which suggests that, during therapy, when using anti-TNF inhibitors, patients should undergo detailed oncological monitoring. On the other hand, we also found that the longer the cells were exposed to the drug, the fold change of the BIRC5 expression was at a level similar to that in fibroblasts that were unstimulated with adalimumab [35]. Further evaluation of the mRNA level of ROCKI during a 24-month follow-up based on the RT-qPCR response indicated that from the 18th month of therapy [M6], the expression of the discussed gene was reduced compared to the previous months and to the controls. One of the causes of the observed pattern of the ROCK1 gene expression might be the lack of an adequate response to treatment, which is not visible at the phenotypic level, based on the PASI and BSA. This underlines the role of the molecular monitoring of the effects of therapy. Chen et al. emphasized the relationship between ROCK 1 and ROCK2 and the JAK-STAT signaling cascade. They indicated that $R O C K 1$ could interact with JAK2 kinase, which results in reduced STAT3 activity in neurons with an elevated leptin concentration in the environment. Thus, ROCKI is closely related to the maintenance of energy homeostasis [36]. Parmentier et al. assessed the effect of a JAK1 inhibitor (upadacitinib) on the levels of ROCK1 and ROCK2. They observed a decrease in the levels of both [37], which may confirm the effectiveness of molecular-targeted therapies. In our work, we also evaluated the expression profile of the RhoA gene. We observed one statistically significant difference between the day before the adalimumab was administered [M0] and the 21st month of 
Table 3 Changes in the dermatological index values: PASI, BSA [\%], DLQI, and DAS28 in the patients with psoriatic arthritis who were being treated with adalimumab during eight time periods (every 3 months)

\begin{tabular}{|c|c|c|c|c|c|c|c|c|c|c|c|c|}
\hline \multirow{2}{*}{$\begin{array}{l}\text { Monitor- } \\
\text { ing }[\mathrm{M}]\end{array}$} & \multicolumn{3}{|l|}{ PASI } & \multicolumn{3}{|c|}{ BSA [\%] } & \multicolumn{3}{|c|}{ DLQI } & \multicolumn{3}{|c|}{ DAS28 } \\
\hline & $\mathrm{Me}$ & Q1 & Q3 & $\mathrm{Me}$ & Q1 & Q3 & $\mathrm{Me}$ & Q1 & Q3 & $\mathrm{Me}$ & Q1 & Q3 \\
\hline 0 & 16.5 & 11.0 & 23.5 & 31.5 & 29.0 & 43.5 & 21.0 & 17.0 & 23.5 & 4.29 & 3.66 & 5.15 \\
\hline 1 & 6.6 & 4.0 & 11.2 & 12.5 & 9.0 & 21.0 & 12.5 & 12.0 & 19.0 & 2.55 & 2.48 & 3.01 \\
\hline 2 & 7.05 & 4.45 & 8.0 & 12.0 & 6.0 & 24.0 & 14.0 & 9.5 & 18.0 & 2.84 & 2.30 & 4.3 \\
\hline 3 & 4.6 & 3.65 & 13.0 & 15.0 & 9.5 & 23.0 & 13.5 & 9.0 & 17.5 & 2.62 & 1.78 & 3.75 \\
\hline 4 & 8.75 & 5.5 & 12.8 & 14.5 & 7.5 & 21.5 & 13.0 & 8.5 & 19.0 & 3.46 & 2.43 & 4.14 \\
\hline 5 & 3.8 & 3.0 & 7.0 & 8.5 & 4.0 & 15.0 & 10.5 & 9.0 & 13.0 & 2.22 & 1.49 & 3.42 \\
\hline 6 & 5.0 & 3.0 & 5.0 & 10.0 & 10.0 & 11.0 & 9.0 & 9.0 & 10.0 & 2.90 & 2.3 & 3.95 \\
\hline 7 & 4.0 & 2.0 & 5.0 & 9.0 & 8.0 & 10.0 & 10.0 & 2.0 & 12.0 & 3.33 & 2.77 & 3.74 \\
\hline 8 & 3.6 & 2.0 & 7.0 & 10.0 & 3.0 & 10.0 & 6.0 & 2.0 & 10.0 & 3.1 & 2.42 & 3.69 \\
\hline
\end{tabular}

$P A S I$ psoriasis area and severity index, $B S A$ body surface area [\%], $D L Q I$ dermatology life quality index, $D A S 28$ disease activity score, $M e$ median, $Q 1$ lower quartile, $Q 3$ upper quartile treatment [M7]. A lower expression of the RhoA gene was found in the patients compared with the controls to the 15th month of treatment [M5], and we next observed a decrease to levels close to those in the healthy group. However, in the 24th month of adalimumab administration [M8], there was a small, statistically insignificant decrease in the activity of the gene. The RhoA/Rho pathway is responsible for determining the balance between the actin polymerization and depolymerization processes, which triggers a caspase cascade. Its role in the biological processes has mainly been described concerning cell migration, proliferation, and apoptosis [38, 39]. In their studies, Li et al. showed the role of RhoA in maintaining the state of homeostasis between the number of Treg lymphocytes and Th17-type lymphocytes [40]. Previous studies indicated that the protein encoded by the RhoA gene interacts in parallel with the MAPK kinases, which are NFkB factors. The overexpression of these three factors was described in rheumatoid arthritis, which indicates that their inhibition would be a promising new therapeutic target [41-43]. Moreover, an analysis that was performed by Deng et al. confirmed the important contribution of RhoA in cartilage destruction and the metabolism of synoviocytes [44]. Also, Kumar et al. and Cho et al. confirmed the role of RhoA in mediating the process of the formation of a network of new blood vessels, as well as with the migration of epithelial cells, which were mediated by the TGF $\beta$ and E-cadherin pathway $[45,46]$.

In their work, Honma et al. evaluated the expression profile of LIMK1 and LIMK2 in biopsied tissue. Although they detected each of these proteins in the other layers of the skin, the profile of their expression was similar. Regarding the expression pattern of LIMKI in the skin biopsies that were obtained from patients suffering from psoriasis and the healthy volunteers, they observed a lower expression of this transcript among the subjects of the study group. They suggested that a negative correlation exists between LIMKI and $M y c$ expression. Concerning the expression of LIMK2, they observed an increase in its expression due to an increase in the concentration of calcium ions in a cell [19]. Taking into account the findings of their study and the role of the increase in the level of calcium ions in the cellular environment in inducing apoptosis [47] to 200-400 nM [48], we can conclude that there is a sequence of actions that increases the mRNA level of LIMK2.

Appleton et al. carried out research on a chondrocyte model. The study indicated the important role of TGF- $\alpha$ in determining the expression and dependence of the Rho/ ROCK/LIMK axis and inductions, including among others the signaling pathways Ras-MEK/ERK and Ras-PI3K, which contribute to the intensification of destructive changes in cartilage [49]. In addition, others have carried out analyses which indicated an inhibition of the Rho/ROCK/LIMK axis expression in different cell types [50, 51]. It has been suggested that not only the existence of a network of complex interactions occurs during the inflammatory process that underlies such conditions as psoriasis [52], but also that there is a need to consider the biological context when interpreting the molecular changes under the influence of various factors [53].

In our studies, we observed that LIMK2 expression was only statistically different between 3rd month of adalimumab therapy [M1] and the 12th month of treatment [M4]. By analyzing the LIMK2 expression profile during the observation, it was found that its expression was at the threshold of detection. A slight increase in the number of copies of the transcript was recorded at the 12th [M3] and 21st months of adalimumab treatment [M7]. The inclusion of ACTB as an endogenous control in the RT-qPCR reaction confirmed the nativity of the RNA extracts and the correct design of the chemical and thermal reaction conditions. For this reason, the LIMK2 expression data that were obtained, depending on the duration of therapy, are reliable and reproducible, despite the relatively low near zero expression. 
Studies have been conducted on the effects of another anti-TNF drug (etanercept) on levels of survival and apoptosis markers. The survivin and caspase 3 genes were used in the analysis. These studies showed an increase in gene expression in both cases. This demonstrates the effect of the anti-TNF drug on the process of apoptosis, which confirms our observations, but also the impact on the process of cell survival [54].

The clinical evaluation of the therapeutic potential of adalimumab was determined using four indicators: PASI, BSA, DAS28, and DLQI [55]. We observed that the 3-month therapy resulted in a decrease in each of the assessed indicators, which underlines the therapeutic effect of adalimumab. However, in the later follow-up period, we recorded an increase in the value of each of the clinical parameters. This confirms that, despite the molecular targeting of adalimumab, this type of therapy also has a drug-resistant effect $[56,57]$. However, understanding the mechanism of action of adalimumab becomes crucial. As a result of studies to indicate the effectiveness of this drug based on the Th17 pathway, it has been proven that the therapeutic effect is caused by turning off the Th17 response [58].

In this study, we also wanted to determine whether there is a correlation between the molecular indicators of the severity of inflammation, including those that are associated with apoptosis, as well as with clinical indicators. The statistical analysis enabled the occurrence of several correlations between gene expression and dermatological index to be observed. In most cases, the described relationship was very strong or almost complete; this was due to the fact that, for the same gene transcript and the different indexes, the direction of the correlation was different (positive, negative). Therefore, the possibility of indicating an adequate lack of response to treatment based only on the RhoA, LIMK2, and ROCK 1 expression patterns was difficult. Moreover, we cannot clearly determine which of the analyzed genes were superior to others. It is possible that increasing the size of the study group would permit similar correlation results to be obtained. Nevertheless, it should also be noted that this does not change the value of molecular research and the search for new molecular systems that can be used in modern diagnostic and therapeutic strategies.

\section{Conclusions}

In conclusion, adalimumab affects the expression profile of the mRNA transcripts that are associated with apoptosis, which is a process that is disturbed in the course of psoriasis. Taking into account the clinical and molecular evaluation, the efficacy of adalimumab in psoriatic arthritis seems to be limited. Therefore, further studies on a large scale investigating the molecular basis for inadequate response to anti-cytokine therapy are worth considering. This study suggests the potential for using changes in the expression profiles of ROCK1, RhoA, and LIMK2, which are responsible for maintaining the balance between proliferation and apoptosis, to evaluate the effectiveness of adalimumab.

Analysis of the expression patterns of ROCKl, RhoA, and LIMK2 genes in patients with psoriatic arthritis, in correlation with values of dermatological indicators, can enrich strategies for treating this disease entity. However, these results should be further confirmed by research conducted at the protein level.

Acknowledgements This study was supported by the Medical University of Silesia in Katowice, Poland, based on decision no. KNW-2I10/D/9/N. This research was supported in part by the PL-Grid Infrastructure. Consent was obtained from the Bioethics Commission of the Medical University of Silesia in Katowice, Poland, no. KNW/0022/ KB1/59/13.

\section{Compliance with ethical standards}

Conflict of interest Authors declare no conflict of interest.

Open Access This article is licensed under a Creative Commons Attribution 4.0 International License, which permits use, sharing, adaptation, distribution and reproduction in any medium or format, as long as you give appropriate credit to the original author(s) and the source, provide a link to the Creative Commons licence, and indicate if changes were made. The images or other third party material in this article are included in the article's Creative Commons licence, unless indicated otherwise in a credit line to the material. If material is not included in the article's Creative Commons licence and your intended use is not permitted by statutory regulation or exceeds the permitted use, you will need to obtain permission directly from the copyright holder. To view a copy of this licence, visit http://creativecommons.org/licenses/by/4.0/.

\section{References}

1. Guo H, Chen L, Cui H, Peng X, Fang J, Zuo Z, et al. Research advances on pathways of nickel-induced apoptosis. Int J Mol Sci. 2016;17:E10.

2. Raj D, Brash DE, Grossman D. Keratinocyte apoptosis in epidermal development and disease. J Invest Dermatol. 2006;126:243-57.

3. Myśliwiec H, Baran A, Flisiak I. Selected aspects of apoptosis in psoriasis. Dermatol Rev. 2017;104:57-63.

4. Nambiar S, Hegde V. Apoptosis in cancer therapy. J Med Radiol Pathol Surg. 2016;3:10-4.

5. Lou H, Pickering M. Extracellular DNA and autoimmune disease. Cell Mol Immunol. 2018;15:746-55.

6. Ray S, Sonthalia N, Kundu S, Ganguly S. Autoimmune disorders: an overview of molecular and cellular basic in today's perspective. J Clin Cell Immunol. 2012;10:3.

7. Sperber K, Beuria P, Singha N, Gelman I, Cortes P, Chen H, et al. Induction of apoptosis by HIV-1-infected monocytic cells. J Immunol. 2003;170(3):1566-78. 
8. Gojniczek K, Jurzak M, Boryka M, Garncarczyk A. Keratosis of the epidermis as an effect of keratinocyte proliferation, differentiation and apoptosis. Pol J Cosmetol. 2007;10(3):146-55.

9. Gordon J, Pound JD. Fortifying B cells with CD154: an engaging tale of many hues. Immunology. 2000;100:269-80.

10. Monaco C, Nanchahal J, Taylor P, Feldmann M. Anti-TNF therapy: past, present and future. Int Immunol. 2015;27(1):55-62.

11. Partyka A, Czopek A, Stanisz-Wallis K, Zagórska A. The use of biopharmaceuticals in the treatment of psoriasis. Post Hig Med Dosw. 2018;72:642-58.

12. Caiazzo G, Fabbrocini G, Di Caprio R, Raimondo A, Scala E, Balato N, et al. Psoriasis, cardiovascular events and biologics: lights and shadows. Front Immunol. 2018;13(9):1668.

13. Balato A, Scala E, Balato N, Caiazzo G, Di Caprio R, Monfrecola $\mathrm{G}$, et al. Biologics that inhibit the Th17 pathway and related cytokines to treat inflammatory disorders. Expert Opin Biol Ther. 2017;17(11):1363-74.

14. Wcisło-Dziadecka D, Grabarek B, Brzezińska-Wcisło L, Mazurek U. Drug resistance in anti-TNF therapy of psoriatic arthritis. Dermatol Rev. 2018;105:625-31.

15. Szepietowski J, Adamski Z, Chodorowska G, Gliński W, Kaszuba A, Placek W, et al. Guidelines of Polish dermatological Society on the treatment of psoriasis vulgaris and arthropathic psoriasis (psoriatic arthritis) with biological drugs. Dermatol Rev. 2010;97:1-3.

16. Sahlol NY, Mostafa MS, Madkour LAE, Salama DM. Low TNFAIP3 expression in psoriatic skin promotes disease susceptibility and severity. PLoS One. 2019;14(5):e0217352.

17. Arul S, Dayalan H, Jegadeesan M, Damonharan P. Induction of differentiation in psoriatic keratinocytes by propylthiouracil and fructose. BBA Clin. 2016;6:82-6.

18. Nowak J, Grzanka A, Żuryń A, Stępień A. The Rho protein family and its role in the cellular cytoskeleton. Post Hig Med Dosw (online). 2008;62:110-7.

19. Honma M, Benitah SA, Watt FM. Role of LIM kinases in normal and psoriatic human epidermis. Mol Biol Cell. 2006;17(4):1888-966.

20. Yang X, Zhu S, Li L, Zhang L, Xian S, Wang Y, et al. Identification of differentially expressed genes and signaling pathways in ovarian cancer by integrated bioinformatics analysis. OncoTargets Ther. 2018;11:1457-74.

21. Ouboussad L, Burska A, Melville A, Buch M. Synovial tissue heterogeneity in rheumatoid arthritis and changes with biologic and targeted synthetic therapies to inform stratified therapy. Front Med (Lausanne). 2019;6:45.

22. Póliska S, Besenyei T, Vegh E, Hamar A, Pusztai A, Vancsa A, et al. Gene expression analysis of vascular pathophysiology related to anti-TNF treatment in rheumatoid arthritis. Arthritis Res Ther. 2019;21(1):94.

23. Dziobek K, Opławski M, Grabarek B, Zmarzły N, Januszyk P, Adwent I, et al. Expression of semaphorin 3B (SEMA3B) in various grades of endometrial cancer. Med Sci Monit. 2019;25:4569-74

24. Lippert J, Appemzeller A, Liang R, Sbiera S, Kircher S, Altieri $\mathrm{B}$, et al. Targeted molecular analysis in adrenocortical carcinomas: a strategy toward improved personalized prognostication. J Clin Endocrinol Metab. 2018;12:4511-23.

25. Tavakolpour S, Darvishi M, Ghasemiadl M. Pharmacogenetics: a strategy for personalized medicine for autoimmune diseases. Clin Genet. 2018;93(3):481-97.

26. Reich A, Adamski Z, Chodorowska G, Kaszuba A, Krasowska D, Lesiak A, et al. Psoriasis. Diagnostic and therapeutic recommendations of the polish dermatological society. Part I: mild psoriasis. Dermatol Rev. 2018;105(2):225-43.

27. Reich A, Szepietowski J, Adamski Z, Chodorowska G, Kaszuba A, Krasowska D, et al. Psoriasis. Diagnostic and therapeutic recommendations of the Polish dermatological society. Part II: moderate to severe psoriasis. Dermatol Rev. 2018;105(3):329-57

28. Batliwalla FM, Li W, Ritchlin CT, Brenner M, Laragione T, Shao T, et al. Microarray analyses of peripheral blood cells identifies unique gene expression signature in psoriatic arthritis. Mol Med. 2005;11(1-12):21-9.

29. Heng YW, Lim HH, Mina T, Utomo P, Zhong S, Lim CT, Koh CG. TPPP acts downstream of RhoA-ROCK-LIMK2 to regulate astral microtubule organization and spindle orientation. J Cell Sci. 2012;125:1579-90.

30. Ren T, Zheng B, Huang Y, Wang S, Bao X, Liu K, et al. Osteosarcoma cell intrinsic PD-L2 signals promotes invasion and metastasis via the RhoA-ROCK-LIMK2 and autophagy pathways. Cell Death Dis. 2019;10(4):261.

31. Julian L, Olson MF. Rho-associated coiled-coil containing kinases (ROCK): structure, regulation, and functions. Small GTPases. 2014;5:e29846.

32. Benitah SA, Valeoron PF, van Aelst L, Matshall CJ, Lacal JC. Rho GTPases in human cancer: an unresolved link to upstream and downstream transcriptional regulation. Biochem Biophys Acta. 2004;1705(2):121-32.

33. Wilkinson S, Peterson HF, Marshall CJ. Cdc42-MRCK and RhoROCK signaling cooperates in myosin phosphorylation and cell invasion. Nat Cell Biol. 2005;7(3):255-61.

34. Zafari P, Rafiei A, Esmaeili SA, Moonesi M, Taghadosi M. Survivin a pivotal antiapoptotic protein in rheumatoid arthritis. J Cell Physiol. 2019;234(2):21575-87.

35. Wcisło-Dziadecka D, Gola J, Grabarek B, Mazurek U, BrzezińskaWcisło L, Kucharz E. Effect of adalimumab on the expression of genes encoding TNF- $\alpha$ signal paths in skin fibroblasts in vitro. Adv Dermatol Allergol. 2018;35(4):413-22.

36. Chen W, Nyuydzefe M, Weiss J, Jingya Z, Waksal S, ZaninZhorov A. ROCK2, but not ROCK1 interact with phosphorylated STAT3 and co-occupies TH17/TFH gene promoters in TH17activated human T cells. Sci Rep. 2018;8:16636.

37. Parmentier J, Voss J, Graff C, Schwartz A, Argiriadi M, Friedman $\mathrm{M}$, et al. In vitro and in vivo characterization of the JAK1 selectivity of upadacitinib (ABT-494). BMC Rheumatol. 2018;2:23.

38. Dasgupta SK, Le A, Vijayan KV, Thiagarajan P. Dasatinib inhibits actin fiber reorganization and promotes endothelial cell permeability through RhoA-ROCK pathway. Cancer Med. 2017;6(4):809-18.

39. Wei F, Liu S, Luo L, Gu N, Zeng Y, Chen X, et al. Anti-inflammatory mechanism of ulinastatin: inhibiting the hyperpermeability of vascular endothelial cells induced by TNF- $\alpha$ via the RhoA/ROCK signal pathway. Int Immunopharmacol. 2017;46:220-7.

40. Li C, Liu PP, Tang DD, Song R, Zhang YQ, Lei S, et al. Targeting the RhoA-ROCK pathway to regulate $\mathrm{T}$-cell homeostasis in hypoxia-induced pulmonary arterial hypertension. Pulm Pharmacol Ther. 2018;50:111-22.

41. Ganesan R, Doss HM, Rasool M. Majoon ushba, a polyherbal compound, suppresses pro-inflammatory mediators and RANKL expression via modulating NFKB and MAPKs signaling pathways in fibroblast-like synoviocytes from adjuvant-induced arthritic rats. Immunol Res. 2016;64(4):1071-86.

42. Liu YR, Yan X, Yu HX, Yao Y, Wang JQ, Li XF, et al. NLRC5 promotes cell proliferation via regulating the NF- $\kappa \mathrm{B}$ signaling pathway in rheumatoid arthritis. Mol Immunol. 2017;91:24-34.

43. Kawanami D, Matova K, Kanazawa Y, Ishizawa S, Yokota T, Utsunomiya K. Thrombin induces MCP-1 expression through Rho-kinase and subsequent $\mathrm{p} 38 \mathrm{MAPK} / \mathrm{NF}-\kappa \mathrm{B}$ signaling pathway activation in vascular endothelial cells. Biochem Biophys Res Commun. 2011;411(4):798-803. 
44. Deng R, Li F, Wu H, Wang WY, Dai L, Zhang ZR, et al. Antiinflammatory mechanism of geniposide: inhibiting the hyperpermeability of fibroblast-like synoviocytes via the RhoA/p38MAPK/ NF-кB/F-actin signal pathway. Front Pharmacol. 2018;9:105.

45. Kumar B, Chile SA, Ray KB, Reddy GE, Addepalli MK, Kumar AS, et al. EGF-C differentially regulates VEGF-A expression in ocular and cancer cells; promotes angiogenesis via RhoA mediated pathway. Angiogenesis. 2011;14(3):371-80.

46. Cho IJ, Kim YW, Han CY, Kim EH, Anderson RA, Lee YS, et al. E-cadherin antagonizes transforming growth factor $\beta 1$ gene induction in hepatic stellate cells by inhibiting RhoA-dependent Smad3 phosphorylation. Hepatology. 2010;52(6):2053-64.

47. Kunzelmann K. Ion channels in regulated cell death. Cell Mol Life Sci. 2016;73(11-12):2387-403.

48. McConkey DJ, Orrenius S. The role of calcium in the regulation of apoptosis. Biochem Biophys Res Commun. 1997;239(2):357-66.

49. Appleton CT, Usmani SE, Mort JS, Beier F. Rho/ROCK and MEK/ERK activation by transforming growth factor- $\alpha$ induces articular cartilage degradation. Lab Invest. 2010;90(1):20-30.

50. Nebl G, Fischer S, Penzel R, Samstag Y. Dephosphorylation of cofilin is regulated through Ras and requires the combined activities of the Ras-effectors MEK and PI3K. Cell Signal. 2004;16(2):235-43.

51. McGonagle D, Lories RJ, Tan AL, Benjamin M. The concept of a "synovio-entheseal complex" and its implications for understanding joint inflammation and damage in psoriatic arthritis and beyond. Arthritis Rheum. 2007;56(8):2482-91.
52. Thomi R, Cazzaniga S, Seyed Jafari SM, Schlapbach C, Hunger RE. Association of hidradenitis suppurativa with T helper 1/T helper 17 phenotypes: a semantic map analysis. JAMA Dermatol. 2018;154(5):592-5.

53. Rianna C, Kumar P, Radmacher M. The role of the microenvironment in the biophysics of cancer. Semir Cell Dev Biol. 2018;73:107-14.

54. Lembo S, Balato N, Caiazzo G, Megna M, Ayala F, Balato A. The effects of etanercept on replication, proliferation, survival and apoptosis markers in moderate to severe psoriasis. JEADV. 2017;31:1-60.

55. Bożek A, Reich A. How to reliably evaluate the severity of psoriasis? Forum Dermatol. 2016;2(1):6-11.

56. Nikolaou M, Pavlopoulou A, Georgakilas AG, Kyrodimos E. The challenge of drug resistance in cancer treatment: a current overview. Clin Exp Metastasis. 2018;35(4):309-18.

57. Bian S, Ferrante M, Gils A. Validation of a drug-resistant antiadalimumab antibody assay to monitor immunogenicity in the presence of high concentrations of adalimumab. AAPS J. 2017;19(2):468-74.

58. Balato A, Schiattarella M, Di Caprio R, Lembo S, Mattii M, Malato N, et al. Effects of Adalimumab therapy in adult subjects with moderate-to-severe psoriasis on Th17 pathway. JEADV. 2014;28:1016-24.

Publisher's Note Springer Nature remains neutral with regard to jurisdictional claims in published maps and institutional affiliations. 